\title{
DISEÑO DE UN CUESTIONARIO PARA MEDIR HABILIDADES DIGITALES DE ESTUDIANTES UNIVERSITARIOS QUE PARTICIPAN DE UN PROGRAMA DE COMPUTACIÓN BASADO EN LA NUBE
}

\author{
Carrasquero, Ender \\ Universidad Euroamericana- UEA \\ Panamá, Panamá \\ ORCID: 0000-0002-9244-0876 \\ López de Ramos, Aura \\ Universidad Internacional de Ciencia y Tecnología - UNICYT \\ Panamá, Panamá \\ ORCID: 0000-0002-8983-9704 \\ León, Mariana \\ Q\&L University \\ Panamá, Panamá \\ ORCID: 0000-0002-0064-9673 \\ Mapp, Ulina \\ ISAE Universidad \\ Panamá, Panamá \\ ORCID: 0000 -0002 -2040- 6724 \\ Reyes, Sebastián \\ Universidad de Santander \\ Panamá, Panamá \\ ORCID: 0000-0002-5824-9832 \\ Suarez, Manning \\ OTEIMA Universidad \\ Panamá, Panamá \\ ORCID: 0000-0003-2740-5748 \\ Montbeliard, Luis \\ Universidad del Istmo \\ Panamá, Panamá \\ ORCID: 0000-0002-0158-3354
}




\author{
Rangel, Victry \\ Universidad Cristiana de Panamá-UCRI \\ Panamá, Panamá \\ ORCID: 0000-0002-6471-9781 \\ De Las Salas, Magdy \\ Universidad Metropolitana de Educación, Ciencia y Tecnología - UMECIT \\ Panamá, Panamá \\ Correo: direccioninvestigacion@umecit.pa \\ Romero, Stephanie \\ Universidad GANEXA \\ Panamá, Panamá \\ ORCID: 0000-0003-4398-6052

\section{Ramos, Erick \\ Panamá, Panamá \\ ORCID: 0000-0002-1173-0143} \\ Universidad Internacional de Ciencia y Tecnología - UNICYT
}

\begin{abstract}
The Association of Private Universities of Panama (AUPPA) with the support of the Secretariat of the Presidency and the Government Innovation Agency (AIG) launched Amazon's AWS Educate Cloud skills training program for Panama. Given the importance, the Research Institute of Private Universities IDIA-AUPPA, decided to develop a study that aims to evaluate the impact of this project This report presents the design and validation of a questionnaire that allows to measure the pre and post level of training in cloud skills of the students participating in the program. The project involves a population made up of 10 private universities and a total of 296 students [1]. The methodology started from a bibliographic review, I designed a battery of questions that responded to 4 dimensions: Possession, Knowledge, Handling of tools and Attitude, twelve indicators and 16 items, based on the model recommended by [2] [3] 4] and [5] The questionnaire was validated by 11 experts; the second version was reduced to 14 questions. The results of the pilot sample according to the total variance, this indicates that 8 components explain $98.207 \%$ of the variance. Its reliability and internal consistency were verified using the Cronbach's Alpha statistic $(\alpha=0.974)$. In conclusion, the results obtained allow us to affirm that this questionnaire is an instrument with an appropriate internal consistency, valid to measure the builder of digital skills in the cloud.
\end{abstract}

Keywords: Desing, Validation, Instrument, Cloud Skills. 


\section{Resumen}

La Asociación de Universidades Particulares de Panamá (AUPPA) con el apoyo de la Secretaría de la Presidencia y la Agencia de Innovación Gubernamental (AIG) lanzó, el programa de formación de habilidades en la Nube para Panamá AWS Educate de Amazon. Dada la importancia, el Instituto de Investigaciones de las Universidades Particulares IDIAAUPPA, decidió desarrollar un estudio que tiene como objetivo evaluar el impacto de este proyecto Este reporte presenta el diseño y validación de un cuestionario que permita medir el nivel pre y post de formación en habilidades en la nube de los estudiantes participantes en el programa. El proyecto involucra a una población constituida por 10 universidades particulares y un total de 296 estudiantes. La metodología partió de una revisión bibliográfica, diseño una batería de preguntas que responde a 4 dimensiones: Posesión, Conocimiento, Manejo de herramientas y Actitud, doce indicadores y 16 ítems, basados en el modelo preconizado por 2] [3] 4] y [5]. El cuestionario, fue validado por 11 expertos la segunda versión quedó reducido a 14 reactivos. Los resultados de la muestra piloto de acuerdo a la varianza total esta indica que 8 componentes explican el 98,207\% de la varianza. Se constató su fiabilidad y consistencia interna mediante el estadístico de Alfa de Cronbach $(\alpha=0.974)$. En conclusión, los resultados obtenidos permiten afirmar que este cuestionario es un instrumento con una consistencia interna apropiada, válido para medir el constructor de habilidades digitales en la nube.

Palabras claves: Diseño, Validación, Instrumento, Habilidades en la nube.

\section{INTRODUCCIÓN}

Como iniciativa de la Asociación Panameña de Universidades Particulares (AUPPA), con el apoyo de la Secretaría de la Presidencia y la Agencia de Innovación Gubernamental (AIG), propiciaron la implementación del Programa AWS Educate de Amazon en Panamá. Como actividad transversal de evaluación el Instituto de Investigaciones de las Universidades Particulares IDIA-AUPPA, asumió la realización de una investigación que tiene como objetivo medir el impacto que este programa tendrá entre los estudiantes de las universidades participantes.

El programa AWS Educate, es un programa auspiciado por Amazon Web Services que tiene como objetivo facilitar el aprendizaje sobre tecnologías de la nube y preparar a los estudiantes para los trabajos del futuro en la nube. El programa consta de Insignias y caminos de carrera en la nube en línea a su propio ritmo, propiciando el aprendizaje práctico con 
crédito promocional de AWS. El programa a nivel mundial a cubierto más de un millón de estudiantes, 3.500 instituciones educativas en más de 200 países [1].

En el caso particular de Panamá, el programa tiene una cobertura de 10 universidades particulares pertenecientes a AUPPA, con una población de 340 estudiantes y 71 docentes.

Con el objeto de poder evaluar el impacto de la iniciativa se diseñó, validó y confiabilizó un instrumento para la medición en una fase pre de las habilidades de los estudiantes en la nube y una fase post morten de las habilidades adquiridas en el programa. Para el diseño del instrumento se realizó una profunda revisión bibliográfica y documental sobre la variable involucrada en el diseño y apegados a los criterios de fondo y forma para la construcción de instrumentos de recolección de información, se elaboró una batería de preguntas que responde a 4 dimensiones: Posesión, Conocimiento, Manejo de herramientas y Actitud, basados en el modelo preconizado para competencias digitales realizado por [2], [3], [4] modelo de adopción de tecnologías de la información y Cloud Computing adaptado por [5]. Posterior a una profunda revisión bibliográfica y documental sobre la variable involucrada en el diseño y apegados a los criterios de fondo y forma para la construcción de instrumentos de recolección de información, se revisó la una batería de preguntas que responde a las mismas 4 dimensiones: doce indicadores y 16 ítems. Esta iniciativa contribuirá a la literatura científica, proporcionado un instrumento válido y confiable para investigadores que necesiten evaluar habilidades en la nube entre poblaciones de estudiantes universitarios.

\section{MÉTODO}

\section{A. Revisión de literatura}

Se realizó una profunda revisión de literatura sobre las variables competencias digitales realizado por [2], [3], [4] y el modelo de adopción de tecnologías de la información y Cloud Computing adaptado por [5] cuyos aportes sirvieron de bases teóricas para la operacionalización de las dimensiones involucradas en el estudio. De esta estructura operacional se diseñó una primera versión del instrumento la cual constó de 4 dimensiones (Posesión, Conocimiento, Manejo de herramientas y Actitud) 12 indicadores y 16 ítems.

\section{B. Síntesis}

La primera versión del instrumento fue objeto de un "focus groups" con los investigadores involucrados a fin de realizar una primera revisión de los ítems. En este mismo sentido, para la segunda sección de trabajo del instrumento, se revisaron que las redacciones cumplieran con los indicadores propuestos.

\section{Revisión por el comité de expertos}

. Continuando con el protocolo de [6] se estableció un Comité de expertos compuesto por 11 profesionales con especialidad en metodología, especialistas en el área de tecnología- y 
evaluación de proyectos educativos de diversos países de Latinoamérica. La propuesta de instrumento AWSPAN a ser validado fue distribuida mediante la plataforma Google Form para facilitar la distribución y la capacidad de emisión de respuestas. Resultado de esta validación de contenido, en el instrumento fueron eliminados dos ítems, resultando un de 14 reactivos.

\section{Prueba de validez de la versión validada}

Según [7] la validez refiere al grado en que un instrumento realmente mide la variable que pretende medir. Por su parte, [8] y [9] reseñan que la validez supone un acuerdo entre el resultado de una prueba o medida y la cosa que se supone medida. Esto significa que, para medir la validez del contenido de los instrumentos de recolección de datos, se debe elaborar una primera versión de cada uno de estos, los cuales deben ser sometidos a juicios de expertos en el área. Para efectos de la validez y confiabilidad del Instrumento AWSPAN, la validez se asumió con la aplicación de la herramienta en una muestra piloto de estudiantes participantes en el programa. La muestra estuvo constituida por diez estudiantes participantes en el programa sin discriminar la universidad de adscripción. Todos los participantes cumplieron la condición de estar activos en el programa AWS Educate y manifestaron su participación voluntaria en el estudio. Los datos demográficos de estos se presentan en la Tabla 1

Tabla 1. Demografía de los participantes

\begin{tabular}{|c|c|c|}
\hline \multirow{2}{*}{ Género } & Masculino & $50 \%(\mathrm{n}=5)$ \\
\cline { 2 - 3 } & Femenino & $50 \%(\mathrm{n}=5)$ \\
\hline \multirow{2}{*}{ Edad } & Rango & $18-25$ años \\
\cline { 2 - 3 } & Media & 22 \\
\hline
\end{tabular}

Fuente: Elaboración propia (2021)

\section{RESULTADOS}

Para determinar la confiabilidad del instrumento de medición "Cuestionario para la medir habilidades digitales de estudiantes universitarios que participan de un programa de computación basado en la nube AWS Educate (AWSPAM)", se aplicó una prueba de niveles, la misma que dio como resultado un coeficiente alfa ( $\alpha=0.9735)$, aplicando prueba de cálculo manual en Excel, con el mismo resultado aplicando SPSS 26 ( $\alpha=0.974$ ), lo cual permite afirmar que el instrumento empleado en la muestra piloto tiene un nivel aceptable de confiabilidad.

Por otra parte, se aplicó una prueba de validez discriminante en escala si se elimina elementos a pesar de haber dado un valor alto. Los resultados reportados no evidencian aumento de la confiabilidad si se elimina un reactivo 
En relación con la matriz varianza total explicada indica que 8 componentes explican el $98,207 \%$ de la varianza. (Tabla-2).

Tabla 2. Varianza total explicada

\begin{tabular}{|c|c|c|c|c|c|c|c|c|c|}
\hline \multicolumn{10}{|c|}{ Varianza total explicada } \\
\hline & \multicolumn{3}{|c|}{ Autovalores iniciales } & \multicolumn{3}{|c|}{ Sumas de extracción de cargas al cuadrado } & \multicolumn{3}{|c|}{ Sumas de rotación de cargas al cuadrado } \\
\hline Componente & Total & \% de varianza & $\%$ acumulado & Total & \% de varianza & \% acumulado & & \% de varianza & \\
\hline 1 & 26.097 & 51.171 & 51.171 & 26.097 & 51.171 & 51.171 & 13.498 & 26.467 & 26.467 \\
\hline 2 & 6.923 & 13.575 & 64.746 & 6.923 & 13.575 & 64.746 & 10.020 & 19.648 & 46.114 \\
\hline 3 & 5.350 & 10.490 & 75.235 & 5.350 & 10.490 & 75.235 & 7.346 & 14.404 & 60.518 \\
\hline 4 & 3.670 & 7.196 & 82.431 & 3.670 & 7.196 & 82.431 & 7.093 & 13.908 & 74.426 \\
\hline 5 & 3.043 & 5.967 & 88.398 & 3.043 & 5.967 & 88.398 & 4.574 & 8.969 & 83.396 \\
\hline 6 & 2.206 & 4.326 & 92.724 & 2.206 & 4.326 & 92.724 & 4.066 & 7.972 & 91.367 \\
\hline 7 & 1.599 & 3.136 & 95.860 & 1.599 & 3.136 & 95.860 & 1.932 & 3.788 & 95.155 \\
\hline 8 & 1.197 & 2.347 & 98.207 & 1.197 & 2.347 & 98.207 & 1.557 & 3.052 & 98.207 \\
\hline 9 & & 1.793 & 100.000 & & & & & & \\
\hline 10 & $1.222 \mathrm{E}-15$ & $2.396 \mathrm{E}-15$ & 100.000 & & & & & & \\
\hline 11 & $8.524 \mathrm{E}-16$ & $1.671 \mathrm{E}-15$ & 100.000 & & & & & & \\
\hline 12 & $7.389 \mathrm{E}-16$ & $1.449 \mathrm{E}-15$ & 100.000 & & & & & & \\
\hline 13 & $6.915 \mathrm{E}-16$ & 1.356E-15 & 100.000 & & & & & & \\
\hline 14 & $5.601 \mathrm{E}-16$ & $1.098 E-15$ & 100.000 & & & & & & \\
\hline 15 & $5.177 E-16$ & $1.015 \mathrm{E}-15$ & 100.000 & & & & & & \\
\hline 16 & $5.001 E-16$ & $9.806 \mathrm{E}-16$ & 100.000 & & & & & & \\
\hline 17 & $4.420 E-16$ & $8.666 \mathrm{E}-16$ & 100.000 & & & & & & \\
\hline 18 & $4.127 E-16$ & $8.092 E-16$ & 100.000 & & & & & & \\
\hline 19 & $3.830 \mathrm{E}-16$ & $7.509 \mathrm{E}-16$ & 100.000 & & & & & & \\
\hline 20 & $3.237 E-16$ & $6.347 \mathrm{E}-16$ & 100.000 & & & & & & \\
\hline 21 & $2.845 \mathrm{E}-16$ & $5.579 \mathrm{E}-16$ & 100.000 & & & & & & \\
\hline 22 & $2.453 \mathrm{E}-16$ & 4.809E-16 & 100.000 & & & & & & \\
\hline 23 & $2.258 \mathrm{E}-16$ & 4.427E-16 & 100.000 & & & & & & \\
\hline 24 & $1.759 \mathrm{E}-16$ & 3.450E-16 & 100.000 & & & & & & \\
\hline 25 & $1.504 \mathrm{E}-16$ & $2.948 E-16$ & 100.000 & & & & & & \\
\hline 26 & $1.259 \mathrm{E}-16$ & $2.468 \mathrm{E}-16$ & 100.000 & & & & & & \\
\hline 27 & $1.056 \mathrm{E}-16$ & $2.070 E-16$ & 100.000 & & & & & & \\
\hline 28 & $5.605 \mathrm{E}-17$ & $1.099 E-16$ & 100.000 & & & & & & \\
\hline 29 & 3.203E-17 & $6.281 \mathrm{E}-17$ & 100.000 & & & & & & \\
\hline 30 & $-5.633 E-20$ & $-1.105 \mathrm{E}-19$ & 100.000 & & & & & & \\
\hline 31 & $-2.519 \mathrm{E}-17$ & $-4.939 \mathrm{E}-17$ & 100.000 & & & & & & \\
\hline 32 & $-3.398 E-17$ & $-6.663 \mathrm{E}-17$ & 100.000 & & & & & & \\
\hline 33 & $-5.970 \mathrm{E}-17$ & $-1.171 \mathrm{E}-16$ & 100.000 & & & & & & \\
\hline 34 & $-9.063 E-17$ & $-1.777 \mathrm{E}-16$ & 100.000 & & & & & & \\
\hline 35 & $-1.280 \mathrm{E}-16$ & $-2.509 \mathrm{E}-16$ & 100.000 & & & & & & \\
\hline 36 & $-1.602 \mathrm{E}-16$ & $-3.141 \mathrm{E}-16$ & 100.000 & & & & & & \\
\hline 37 & $-2.017 \mathrm{E}-16$ & $-3.954 \mathrm{E}-16$ & 100.000 & & & & & & \\
\hline 38 & $-2.284 \mathrm{E}-16$ & $-4.479 E-16$ & 100.000 & & & & & & \\
\hline 39 & $-2.641 \mathrm{E}-16$ & $-5.179 \mathrm{E}-16$ & 100.000 & & & & & & \\
\hline 40 & $-3.013 \mathrm{E}-16$ & $-5.907 \mathrm{E}-16$ & 100.000 & & & & & & \\
\hline 41 & $-3.096 \mathrm{E}-16$ & $-6.071 \mathrm{E}-16$ & 100.000 & & & & & & \\
\hline 42 & $-3.390 E-16$ & $-6.647 E-16$ & 100.000 & & & & & & \\
\hline 43 & $-3.666 \mathrm{E}-16$ & $-7.188 \mathrm{E}-16$ & 100.000 & & & & & & \\
\hline 44 & $-4.343 E-16$ & $-8.516 \mathrm{E}-16$ & 100.000 & & & & & & \\
\hline 45 & $-4.565 E-16$ & $-8.952 E-16$ & 100.000 & & & & & & \\
\hline 46 & $-4.959 \mathrm{E}-16$ & $-9.723 E-16$ & 100.000 & & & & & & \\
\hline 47 & $-5.447 E-16$ & $-1.068 \mathrm{E}-15$ & 100.000 & & & & & & \\
\hline 48 & $-6.495 E-16$ & $-1.274 \mathrm{E}-15$ & 100.000 & & & & & & \\
\hline 49 & $-7.947 \mathrm{E}-16$ & $-1.558 \mathrm{E}-15$ & 100.000 & & & & & & \\
\hline 50 & $-9.120 E-16$ & $-1.788 \mathrm{E}-15$ & 100.000 & & & & & & \\
\hline 51 & $-1.206 \mathrm{E}-15$ & $-2.365 \mathrm{E}-15$ & 100.000 & & & & & & \\
\hline
\end{tabular}

Por otra parte, se estableció, la correlación entre las cuatro dimensiones a través de la prueba Pearson sobre la cual se reporta para la dimensión Posesión, Conocimiento, Manejo, Actitud obteniéndose datos que se presentan en la tabla 3.

Tabla 3. Correlación Pearson

\begin{tabular}{l|c|c|c|c}
\cline { 2 - 5 } & POSESIÓN & CONOCIMIENTO & MANEJO & ACTITUD \\
\hline POSESIÓN & 1 & & & \\
\hline CONOCIMIENTO & .722 & 1 & & \\
\hline MANEJO & .803 & .909 & 1 & \\
\hline ACTITUD & .722 & .781 & .792 & 1 \\
\hline
\end{tabular}


Los pesos obtenidos dentro de las dimensiones son suficientes $(<0.4)$, entendiendo de que existe correlación con pesos mayores a 0.4., obtenido en todas las dimensiones con una importante carga o valores a la hora de interpretar los resultados obtenidos.

\section{CONCLUSIONES}

Los resultados obtenidos en el análisis de fiabilidad permiten afirmar que este cuestionario es un instrumento con una consistencia interna apropiada, válido para medir el constructor de habilidades digitales en la nube, lo que permite concluir que el cuestionario diseñado se ajusta al modelo propuesto en sus cuatro dimensiones.

No obstante, se es conscientes de que el instrumento AWSPAN, presenta las limitaciones de que, al ser destinado a un proceso de autoevaluación de estudiantes sobre un proceso de habilidades digitales en la nube, la información recabada con el mismo, pueda presentar sesgos propios de todo proceso subjetivo, pudiendo influir esto, en alguna medida los resultados.

\section{Referencias}

[1] Amazon Web Service, Informe interno del Programa AWS Educate Panamá.2021.

[2] MARTín, Sonia Casillas, et al. Estudio psicométrico de un cuestionario para medir la competencia digital de estudiantes universitarios (CODIEU). Education in the knowledge society (EKS), 2018, vol. 19, no 3, p. 61-81.

[3] CERVERA, Mercè Gisbert; VIDAL, Cinta Espuny; MARTÍNEZ, Juan González. INCOTIC. Una herramienta para la@ utoevaluación diagnóstica de la competencia digital en la universidad. Profesorado. Revista de Currículum y Formación de Profesorado, 2011, vol. 15, no 1, p. 75-90..

[4] J. Ramírez, F. González, y A. López, Desarrollo de un instrumento de recolección de datos para la evaluación del nivel de alfabetización digital de estudiantes universitarios, Rev-RIC, vol. 5, n. 2, pp. 59-71, mar. 2020. https://doi.org/10.33412/rev-ric.v5.2.2506

[5] PALOS-SANCHEZ, Pedro; REYES-MENENDEZ, Ana; SAURA, Jose Ramon. Modelos de Adopción de Tecnologías de la Información y Cloud Computing en las Organizaciones. Información tecnológica, 2019, vol. 30, no 3, p. 3-12. Beaton

[6] Hernandez BEATON, Dorcas E., et al. Guidelines for the process of cross-cultural adaptation of selfreport measures. Spine, 2000, vol. 25, no 24, p. 3186-3191.

[7] HERNÁNDEZ, Fernández. Batista,(2010) Metodología de la Investigación. Editorial Mac Graw Hill. México, vol. 2.

[8] TAMAYO, Mario. Metodología de la Investigación. México: Limusa, 2007.

[9] CARRASQUERO, Ender. Adaptación y validación española del instrumento de percepción Cornell Musculoskeletal Discomfort Questionnaires (CMDQ). Desarrollo gerencial, 2015, vol. 7, no 2, p. 3646. 


\section{Autorización y Licencia CC}

Los autores autorizan a APANAC XVIII a publicar el artículo en las actas de la conferencia en Acceso Abierto (Open Access) en diversos formatos digitales (PDF, HTML, EPUB) e integrarlos en diversas plataformas online como repositorios y bases de datos bajo la licencia CC:

Attribution-NonCommercial-ShareAlike 4.0 International (CC BY-NC-SA 4.0) https://creativecommons.org/

licenses/by-nc-sa/4.0/.

Ni APANAC XVIII ni los editores son responsables ni del contenido ni de las implicaciones de lo expresado en el artículo. 